\title{
Normalization of coronary microvascular reactivity and improvement in myocardial perfusion by surgical vascular endothelial growth factor therapy combined with oral supplementation of L-arginine in a porcine model of endothelial dysfunction
}

Pierre Voisine, MD, ${ }^{a}$ Cesario Bianchi, MD, PhD, ${ }^{a}$ Tanveer A. Khan, MD, ${ }^{a}$ Marc Ruel, MD, MPH, ${ }^{\text {a }}$ Shu-Hua Xu, PhD, ${ }^{\text {a }}$ Jun Feng, MD, PhD, ${ }^{a}$ Jian Li, MD, PhD, ${ }^{b}$ Tamer Malik, MD, ${ }^{a}$ Audrey Rosinberg, MD, a and Frank W. Sellke, MD ${ }^{a}$

From the Divisions of Cardiothoracic Surgery and Cardiology, ${ }^{\mathrm{b}}$ Beth Israel Deaconess Medical Center, Boston, Mass.

Supported by grant R01 HL69024 from the National Institutes of Health (Dr Sellke). Dr Voisine is a research fellow of the Heart and Stroke Foundation of Canada.

Read at the Eighty-fourth Annual Meeting of The American Association for Thoracic Surgery, Toronto, Ontario, Canada, April 25-28, 2004.

Received for publication April 24, 2004; revisions received Nov 15, 2004; accepted for publication Dec 7, 2004.

Address for reprints: Pierre Voisine, MD, Division of Cardiothoracic Surgery, Beth Israel Deaconess Medical Center, 330 Brookline Ave, DANA 881, Boston, MA 02215 (E-mail: pvoisine@bidmc.harvard.edu).

J Thorac Cardiovasc Surg 2005;129:1414-20 $0022-5223 / \$ 30.00$

Copyright $\odot 2005$ by The American Association for Thoracic Surgery

doi:10.1016/j.jtcvs.2004.12.046
Objective: Vascular endothelial growth factor acts in part through nitric oxide release, the availability of which is decreased in endothelial dysfunction associated with advanced coronary artery disease. This could explain the relatively disappointing results of vascular endothelial growth factor therapy in clinical studies compared with animal studies. We examined the influence of L-arginine supplementation to vascular endothelial growth factor therapy on myocardial microvascular reactivity and perfusion in a porcine model of endothelial dysfunction.

Methods: Twenty-four pigs were fed either a normal (NORM, $\mathrm{n}=8$ ) or highcholesterol diet with (CHOL-ARG, $\mathrm{n}=8$ ) or without $(\mathrm{CHOL}, \mathrm{n}=8)$ L-arginine. All pigs underwent ameroid placement on the circumflex artery and then 3 weeks later received surgical vascular endothelial growth factor treatment. Four weeks after treatment, endothelial-dependent coronary microvascular responses and lateral myocardial perfusion were assessed. Endothelial cell density was determined by means of immunohistochemistry. Vascular endothelial growth factor, endothelial nitric oxide synthase, and Akt levels were determined by means of immunoblotting.

Results: Pigs from the CHOL group showed endothelial dysfunction in the circumflex territory, which was normalized by L-arginine supplementation. Vascular endothelial growth factor treatment was ineffective in the CHOL group (circumflex/ left anterior descending coronary artery blood flow ratios: 0.95 [rest] and 0.74 [pace] before-after treatment; $P<.05$ compared with the NORM group). Addition of L-arginine restored the angiogenic effect of vascular endothelial growth factor (ratios: 1.13 [rest] and 1.20 [pace]; $P<.05$ ) and was associated with increased endothelial cell density, as well as vascular endothelial growth factor, endothelial nitric oxide synthase, and Akt protein levels in the ischemic territory.

Conclusions: L-Arginine supplementation can restore normal endothelium-dependent vasorelaxation and angiogenic response to vascular endothelial growth factor in a swine model of chronic myocardial ischemia with hypercholesterolemia-induced endothelial dysfunction. These findings suggest a putative role for L-arginine in combination with vascular endothelial growth factor therapy for end-stage coronary artery disease.

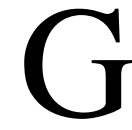
rowth factor-based therapeutic angiogenesis is an attractive alternative strategy for patients with advanced coronary artery disease (CAD) who are not suitable candidates for conventional revascularization techniques. Despite some encouraging results in clinical trials testing the efficacy of vascular endothelial growth factor (VEGF) and fibroblast growth factor (FGF) 2, the use of growth factors in human 
subjects has not fulfilled the enthusiastic expectations that successful studies in several animal models of ischemia have generated. ${ }^{1,2}$

The reasons for the discrepancies between the results from animal and human studies are unclear. However, VEGF and other angiogenic growth factors operate in large part through the release of endothelial-derived nitric oxide (NO) through the activation of tyrosine kinase receptors. ${ }^{3}$ Because patients with CAD typically display several risk factors for endothelial dysfunction, the lack of growth factor-mediated angiogenic effects in this population might be related to a deficiency in the stimulated release of $\mathrm{NO}$ as a result of their disease state. ${ }^{4}$ There is in vitro evidence that an important relationship exists between the release of NO and the regulation of VEGF-mediated blood vessel growth and development. VEGF enhances the expression of endothelial nitric oxide synthase (eNOS) in native and cultured endothelial cells, an effect that might be important in the process of VEGF-induced angiogenesis, ${ }^{5}$ and eNOS expression is increased in proliferating compared with confluent endothelial cells. ${ }^{6}$ Moreover, inhibitors of NOS suppress angiogenesis, and the proliferative effect of VEGF is decreased in the presence of NOS inhibitors. ${ }^{7,8}$ A recent study also shows in vivo inhibition of VEGF-mediated myocardial angiogenesis in a porcine model of hypercholesterolemia-induced endothelial dysfunction. ${ }^{9}$

Meanwhile, a considerable number of animal and human studies suggest a beneficial role of L-arginine, the substrate for eNOS, on endothelial function. L-Arginine has been shown to prevent intimal thickening in the coronary arteries of hypercholesterolemic rabbits independently of alterations in plasma cholesterol levels. ${ }^{10}$ L-Arginine administration in human subjects has been associated with improved epicardial and microvascular responses to acetylcholine in patients with endothelial dysfunction. ${ }^{11}$ In a double-blind, randomized study investigating the effects of chronic dietary supplementation with L-arginine in human subjects with nonobstructive $\mathrm{CAD}$, an increase in coronary blood flow in response to acetylcholine (associated with a decrease in plasma endothelin concentrations and an improvement in patients' symptoms) was observed. ${ }^{12}$

Accordingly, we examined the effects of chronic oral administration of L-arginine on the impaired angiogenic response to VEGF in a porcine model of hypercholesterolemia-induced endothelial dysfunction.

\section{Methods \\ Study Design}

Twenty-four Yucatan miniswine (Sinclair Research, Columbia, Mo) were either fed a regular chow (NORM, $n=8)$ or a hypercholesterolemic diet $(\mathrm{n}=16)$ with (HICHOL-ARG, $\mathrm{n}=8$ ) or without (HICHOL, $\mathrm{n}=8$ ) oral supplementation of $\mathrm{L}$-arginine (50 $\mathrm{mg} / \mathrm{kg}$ by mouth twice daily). The high-cholesterol diet (4\% cholesterol, $17.2 \%$ coconut oil, $2.3 \%$ corn oil, $1.5 \%$ sodium cholate, and $75 \%$ regular chow) and L-arginine administration were started at 7 weeks of age and continued throughout the entire study period (total period of 20 weeks). All animals received humane care in compliance with the Harvard Medical Area Institutional Animal Care and Use Committee and the National Research Council's "Guide for the Care and Use of Laboratory Animals" (National Institutes of Health publication no. 5377-3, revised 1996). The remaining steps of the protocol have been described elsewhere ${ }^{9}$ and are briefly outlined below.

An ameroid constrictor (1.75-mm internal diameter) was placed on the proximal circumflex coronary artery of each pig through a left minithoracotomy. Red-gold microspheres (BioPhysics Assay Laboratory, Worcester, Mass) were injected in the left atrium during temporary occlusion of the circumflex artery to determine the exact myocardial territory at risk (the shadow-labeling procedure).

Three weeks after ameroid insertion, coronary angiograms were recorded, and a left thoracotomy was performed. Microspheres $\left(1.5 \times 10^{7}\right)$ were injected both at rest (samarium) and during pacing at 150 beats/min (lanthanum) for perfusion analysis. In all pigs VEGF administration was then started with an osmotic pump system (ALZET, model 2MLA; Alza, Palo Alto, Calif), delivering a total of $2 \mu \mathrm{g}$ of human recombinant $\mathrm{VEGF}_{165}$ (R\&D Systems, Minneapolis, Minn) at a rate of $3 \mathrm{ng} / \mathrm{h}$ within the myocardium adjacent to the circumflex artery.

Four weeks later, coronary arteriography was repeated, hearts were exposed, and microspheres (lutetium at rest and ytterbium under pacing) were injected as described above. After death, hearts were harvested and cut at the midventricular level and then sectioned into systematically identified segments. Samples from the anterior and left lateral walls were used for molecular studies, in vitro assessment of microvessel reactivity, and microsphere perfusion analyses.

\section{In Vitro Assessment of Coronary Microvessel Reactivity}

After cardiac harvest, epicardial coronary arterioles (70-150 $\mu \mathrm{m}$ in diameter and 1-2 $\mathrm{mm}$ in length) originating from branches of the left anterior descending artery (LAD) and left circumflex artery were dissected and examined in isolated organ chambers, as described previously. ${ }^{13}$ The responses to sodium nitroprusside (SNP; $1 \mathrm{nmol} / \mathrm{L}$ to $100 \mu \mathrm{mol} / \mathrm{L}$ ), an endotheliumindependent cyclic guanosine monophosphate-mediated vasodilator, as well as responses to adenosine $5^{\prime}$ diphosphate (ADP; $(1 \mathrm{nmol} / \mathrm{L}$ to $10 \mu \mathrm{mol} / \mathrm{L}$ ) and VEGF ( $1 \mathrm{fmol} / \mathrm{L}$ to $1 \mathrm{nmol} / \mathrm{L})$, endothelium-dependent receptor-mediated vasodilators that act through bioavailable NO, were studied. ${ }^{14}$

\section{Perfusion Analyses}

Myocardial blood flows were determined during each procedure with isotope-labeled microspheres (BioPAL, Worcester, Mass) by using methods previously reported. ${ }^{9,15}$ Isotope-labeled microspheres of different isotopic masses were used at each experimental stage, as explained in the "Study design" section.

\section{Western Blotting}

Whole-cell lysates were isolated from the homogenized anterior and lateral myocardium, and Western Blot analyses were per- 
formed as previously described ${ }^{9}$ with a 1:1000 dilution of polyclonal anti-human VEGF antibody (Oncogene, Boston, Mass), a 1:1000 dilution of anti-eNOS antibody (Cell Signaling, Beverly, Mass), and a 1:1000 dilution of polyclonal anti-rabbit antibody for Akt (Santa Cruz Biotechnology Inc, Santa Cruz, Calif).

\section{Immunohistochemistry}

Staining with CD31 antibody (BD Biosciences Pharmingen, San Diego, Calif) was done according to methods previously reported. ${ }^{9}$

\section{Regional Contractility}

Regional myocardial function was assessed by means of sonomicrometry (Sonometrics Digital Ultrasonic Measurement System; Sonometrics Corp, London, Ontario, Canada) with 2 digital piezoelectric ultrasonic probes $(2.0 \mathrm{~mm})$ implanted in the subendocardial layer approximately $10 \mathrm{~mm}$ apart within the collateral-dependent area parallel to the minor axis of the heart and by determining the percentage of segmental shortening during systole.

\section{Data Analysis}

Data are reported as means \pm standard error of the mean. Comparisons between groups were analyzed by means of 1-way analysis of variance with the Bonferroni multiple comparison test, followed by a 2-tailed $t$ test with Microsoft Excel (Microsoft Corp, Seattle, Wash) and GraphPad Prism (GraphPad Software Inc, San Diego, Calif). Microvessel responses are expressed as the percentage of relaxation of the preconstricted diameter and were analyzed by fitting a linear regression model examining the relationship among vessel relaxation, log concentration of the vasoactive agent of interest, and the experimental group or myocardial territory of origin (SAS Version 8, Cary, NC). Immunoblottings were analyzed as previously described. ${ }^{9}$

\section{Results}

\section{Animal Model}

Two pigs from the HICHOL group died before completion of the experimental protocol. The first one died 24 hours after ameroid placement, probably from arrhythmia, and the second pig died at the time of the treatment procedure from acute heart failure after pacing at 150 beats/min, which eventually led to ventricular fibrillation that was unresponsive to pharmacologic intervention and defibrillation. There was one death in the HICHOL-ARG group 3 days after VEGF treatment caused by acute aortic thrombosis at the iliac bifurcation level. All pigs from the NORM group completed the study.

Serum cholesterol levels were not significantly different in the HICHOL and HICHOL-ARG groups but were significantly higher than in the NORM pigs at all time points. Total serum cholesterol levels for these 3 groups were, respectively, $9.3 \pm 1.6,10.0 \pm 0.9$, and $1.1 \pm 0.2 \mathrm{mmol} / \mathrm{L}$ at the time of ameroid placement; $10.2 \pm 2.1,9.3 \pm 1.0$, and $1.0 \pm 0.8 \mathrm{mmol} / \mathrm{L}$ at the time of VEGF treatment; and 10.4 $\pm 1.3,10.1 \pm 1.2$, and $1.2 \pm 0.2 \mathrm{mmol} / \mathrm{L}$ at the time of harvest $(P<.01)$.
L-Arginine levels were not statistically different in the 3 groups (HICHOL, $62.6 \pm 9.2 \mathrm{mmol} / \mathrm{mL}$; HICHOL-ARG, $68.3 \pm 8.6 \mathrm{mmol} / \mathrm{mL}$; and NORM, $66.8 \pm 9.8 \mathrm{mmol} / \mathrm{L}$ ).

Coronary angiograms confirmed the complete closure of the ameroid constrictors in all animals, with impaired distal filling of the circumflex (TIMI 2 flow). There was no significant difference in the visible collateral vessel development among the 3 groups.

\section{Microvessel Reactivity}

Figure 1 shows the relaxation curves to increasing concentrations of vasodilators after preconstriction with the thromboxaneprostaglandin endoperoxide analogue U46619. Endothelial dysfunction in the high-cholesterol-diet group was evidenced by the significantly impaired vasorelaxation to ADP and VEGF, 2 endothelium-dependent vasodilators, in the circumflex distribution $(P<.001$ and $P=.006$ vs the LAD territory, respectively; Figure 1,B). Relaxation to the endotheliumindependent agent SNP also showed a decreased response in the ischemic area $(P=.03$; Figure $1, B)$. Contrastingly, there was no difference in the curve responses from the circumflex and LAD territories in the normal diet group (Figure 1, A), and all the altered microvascular responses induced by the highcholesterol diet were normalized by L-arginine supplementation (Figure 1, C).

\section{Endothelial Cell Density}

Figure 2 shows the density of $\mathrm{CD} 31^{+}$capillary endothelial cells in the left lateral wall of pigs from all diet groups 4 weeks after initiating VEGF treatment. The cell density was significantly higher in the normal diet and the L-arginine groups compared with that in the high-cholesterol-diet group ( $95 \pm 12$ vs $90 \pm 15$ vs $48.3 \pm 10$, respectively; $P<$ $.05)$. Examples of histologic sections are also shown.

\section{Regional Contractility}

For differences in the posttreatment versus pretreatment percentages of segmental shortening in the short axis, although a trend toward increased improvements in the normal diet $(7.0 \% \pm 2.5 \%)$ and the L-arginine $(7.9 \% \pm 3.3 \%)$ groups compared with that in the high-cholesterol group $(1.3 \% \pm 0.8 \%)$ was shown, statistical significance was not achieved because of variability within the groups $(P=.21$ by means of 1-way analysis of variance; $P=.08$ for normal diet vs high-cholesterol diet; $P=.09$ for L-arginine vs high-cholesterol diet).

\section{Myocardial Perfusion}

The results of isotope-labeled microsphere assays are depicted in Figure 3. Three weeks after ameroid placement, at the time of VEGF treatment initiation, baseline circumflex coronary flow was similar among the 3 groups, both at rest (NORM, $0.53 \pm 0.05 \mathrm{~mL} \cdot \mathrm{min}^{-1}$. $\mathrm{g}^{-1}$; HICHOL, $0.51 \pm 0.07 \mathrm{~mL} \cdot \mathrm{min}^{-1} \cdot \mathrm{g}^{-1}$; HICHOL- 


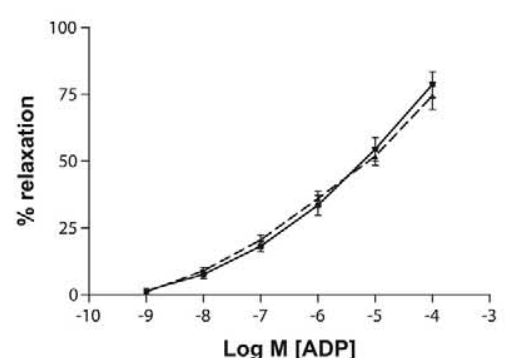

\section{A: Normal diet}

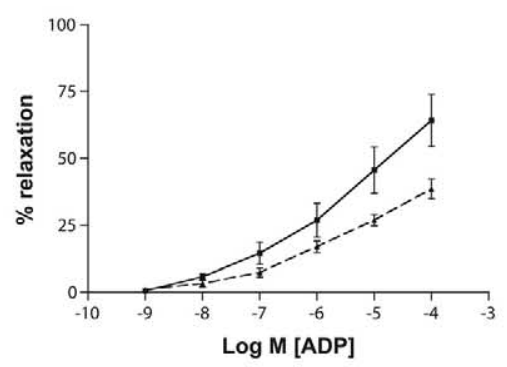

\section{B: High-cholesterol diet}

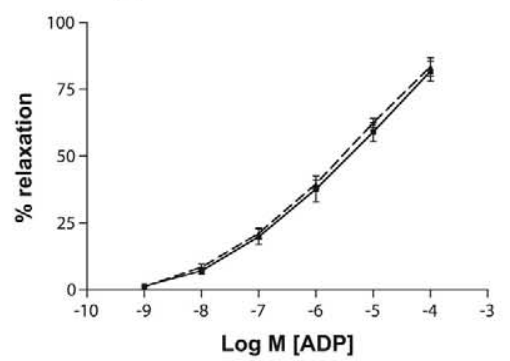

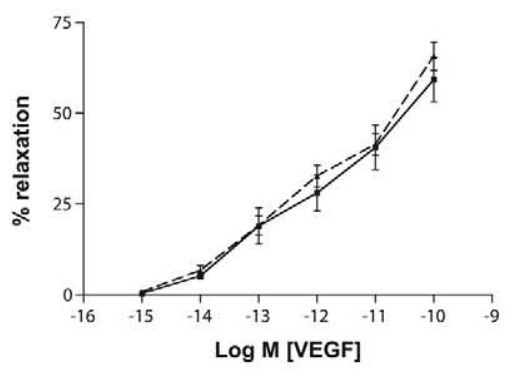
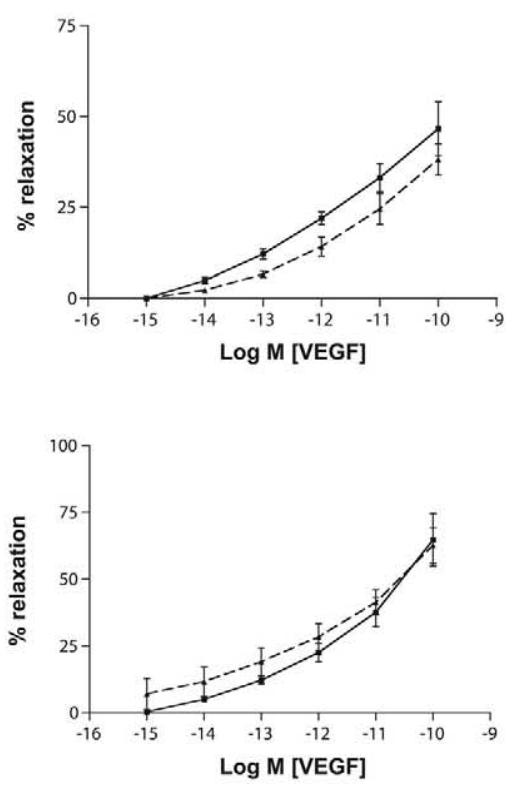
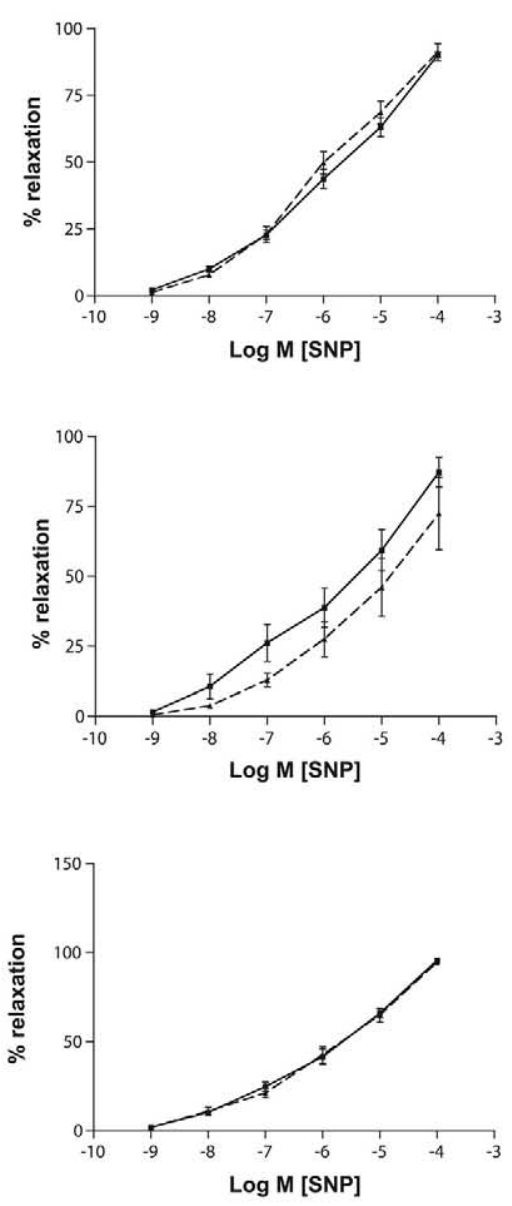

LAD

Figure 1. Microvascular reactivity studies: percentage relaxation to increasing concentrations of vasodilating agents after preconstriction with U46619. ADP, Endothelium-dependent vasodilator adenosine diphosphate; VEGF, partially endothelium-dependent vasodilator vascular endothelial growth factor; SNP, sodium nitroprusside, an endothelium-independent vasodilator. A, Normal diet group. B, High-cholesterol diet group. C, High-cholesterol diet with L-arginine supplementation.

ARG, $0.47 \pm 0.03 \mathrm{~mL} \cdot \min ^{-1} \cdot \mathrm{g}^{-1}$ ) and during pacing (NORM, $0.49 \pm 0.06 \mathrm{~mL} \cdot \mathrm{min}^{-1} \cdot \mathrm{g}^{-1}$; HICHOL, 0.49 $\pm 0.04 \mathrm{~mL} \cdot \mathrm{min}^{-1} \cdot \mathrm{g}^{-1}$; HICHOL-ARG, $0.45 \pm 0.05$ $\left.\mathrm{mL} \cdot \min ^{-1} \cdot \mathrm{g}^{-1}\right)$. After 4 weeks of VEGF therapy, at the time of the final procedure, circumflex myocardial blood flow both at rest and during pacing was significantly higher in the normal diet group than in its high-cholesterol counterpart. Respective ratios of blood flow in the circumflex to the LAD territories in the posttreatment versus pretreatment settings were $1.18 \pm 0.07$ (normal diet) versus $0.95 \pm 0.03$ (high-cholesterol diet) at rest $(P$ $<.05$ ) and $1.13 \pm 0.05$ (normal diet) versus $0.74 \pm 0.08$ (high-cholesterol diet) during pacing $(P<.01)$, thus corresponding in increases from baseline perfusion of
$18 \%$ and $13 \%$ for the normal diet group, respectively, at rest and during pacing, whereas there were decreases of $5 \%$ at rest and $20 \%$ during pacing for the high-cholesterol group. L-Arginine administration resulted in improvements in perfusion that were similar to those observed in the normal diet group (ratios of $1.13 \pm 0.05$ at rest and $1.20 \pm 0.12$ during pacing; not significant vs NORM, $P$ $<.05$ vs HICHOL).

\section{Western Blot Analyses}

Myocardial VEGF, eNOS, and Akt protein expression were significantly different among the 3 groups. Densitometry of protein levels in the ischemic and the nonischemic territories is displayed in Figure 4. 


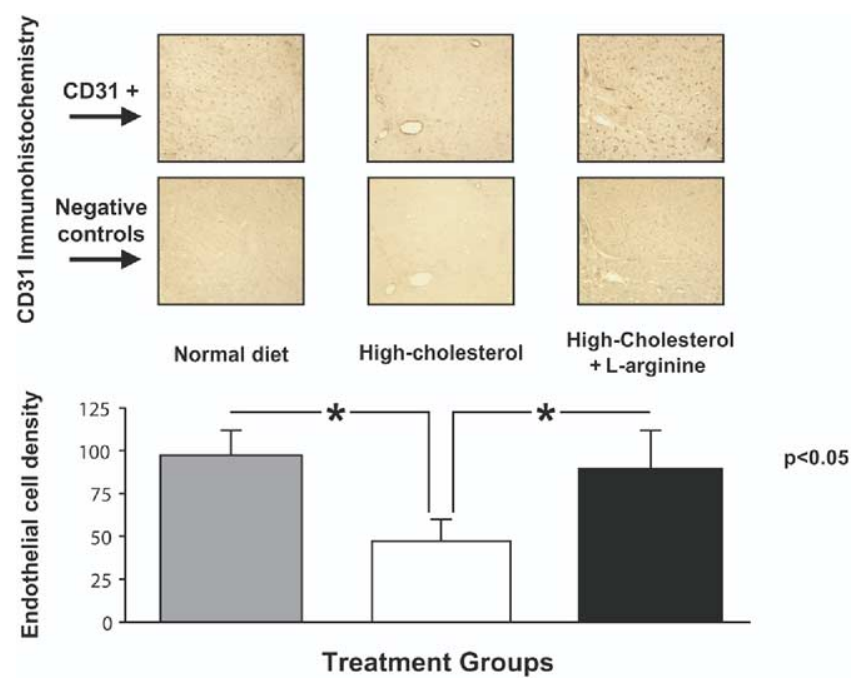

Figure 2. Top, Immunohistochemical staining for CD31 $\left(C D 31^{+}\right)$ and corresponding negative control of an adjacent section (negative controls) in the left circumflex territory according to diet regimen 4 weeks after VEGF treatment. Bottom, Bar graph summarizing endothelial cell density results in the corresponding diet groups. ${ }^{*} P<.05$.

\section{Discussion}

In this study significant coronary microvessel endothelial dysfunction was induced in a swine model of chronic ischemia by means of administration of a cholesterol-rich diet. This was associated with a markedly decreased functional response to the angiogenic effects of exogenous VEGF in comparison with that seen in pigs undergoing the same VEGF treatment but maintained on a regular chow. Chronic oral supplementation of L-arginine to pigs receiving the same high-cholesterol regimen normalized their coronary microvascular response to endothelium-dependent and endothelium-independent vasorelaxing agents, which was

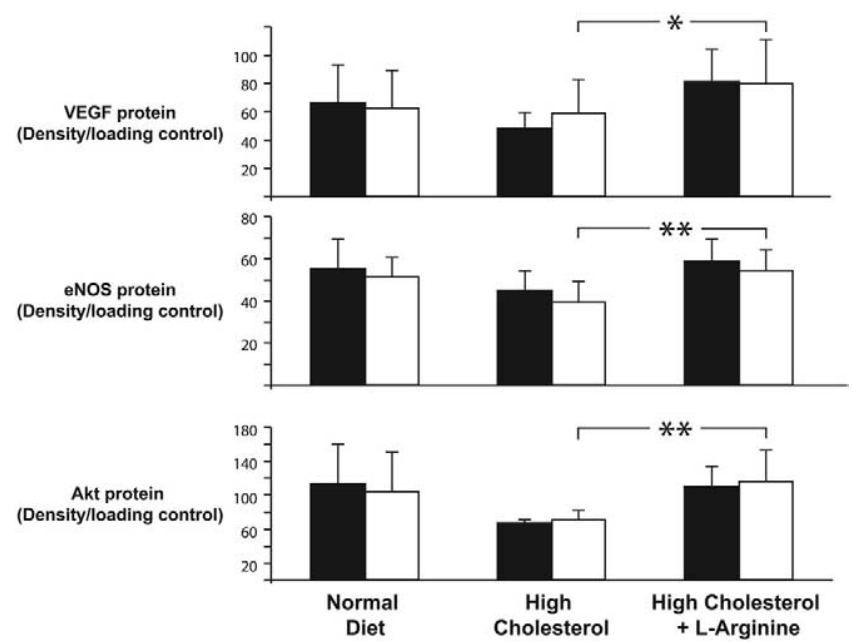

Figure 4. A, Western blot analysis of VEGF, eNOS, and Akt protein levels in myocardial tissue harvested from the ischemic and nonischemic territories in all pigs 4 weeks after VEGF treatment. Ponceau $S$ optical density was used as a correction factor for quantification. Relevant statistically significant differences in expression levels were highlighted, and $\boldsymbol{P}$ values are presented. Filled column, LAD; open column, left circumflex artery. ${ }^{*} P<.05$; ${ }^{* *} \boldsymbol{P}<.01$.

associated with a restored angiogenic response to exogenous VEGF.

Porcine models of hypercholesterolemia-induced endothelial dysfunction have previously been described. Pigs fed a cholesterol-rich diet for as little as 9 weeks have been shown to display attenuated endothelium-dependent relaxation to serotonin, despite the absence of intimal proliferative changes, ${ }^{16}$ and 10 weeks of this diet has also been associated with reduced vasorelaxation to bradykinin. ${ }^{17} \mathrm{Al}-$ though pigs from our study clearly showed impaired vasorelaxation to ADP and VEGF, both endothelium-dependent

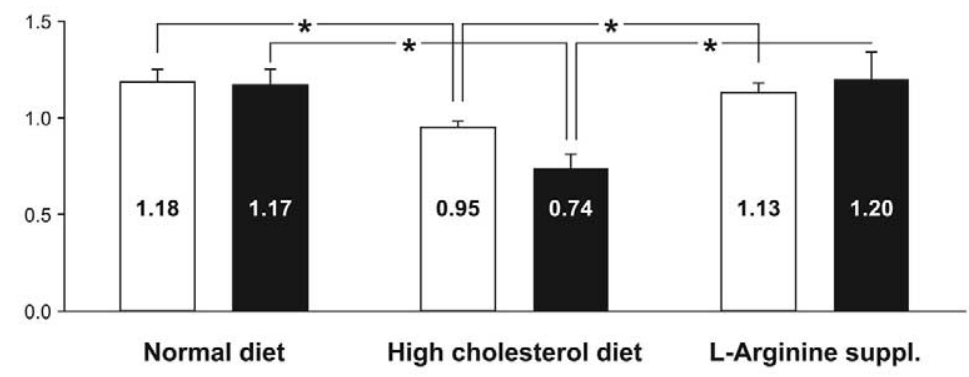

1418
Figure 3. Perfusion analyses. Post-VEGF versus preVEGF treatment ratios of circumflex (LCX) versus LAD blood flows in the different group treatments. The ratios are presented for both resting and stress conditions by ventricular pacing at 150 beats/min. Open column, Rest; filled column, pace. ${ }^{*} P<.05$. 
vasodilators, there also was a significantly decreased response to SNP, an endothelium-independent agent. This suggests a partial contribution from the smooth muscle cell-mediated vasodilatation to the observed response in our model, a function that might be directly affected by progression of the atherosclerotic process in the microcirculation. Although endothelium independent, SNP still ultimately causes vasorelaxation through $\mathrm{NO}$ release. The altered response in the high-cholesterol group could be explained in part by an accelerated degradation of SNPderived NO by the increased superoxide anion production associated with hypercholesterolemia. Increased oxidative stress resulting from the induction of xanthine oxidase and reduced nicotinamide adenine dinucleotide/reduced nicotinamide adenine dinucleotide phosphate oxidase and uncoupling of eNOS with inactivation from reactive oxygen species have been proposed to explain the relationship between hypercholesterolemia and endothelial dysfunction. ${ }^{18}$ In our study the normalization of the microvascular response to ADP and VEGF resulting from L-arginine administration was associated with significant increases in levels of VEGF, Akt, and eNOS. Although the mechanisms that govern the activation of the signaling pathways leading to Akt and eNOS expression remain incompletely understood, these results suggest that modulation of expression of these molecules plays a role in the endothelial dysfunction that accompanies hypercholesterolemia. Asymmetric dimethylarginine, an arginine analog that competes with L-arginine for eNOS, ${ }^{19}$ is increased in hypercholesterolemia and can also contribute to impairment of endothelial function. ${ }^{20}$ Providing more substrate for eNOS in our HICHOL-ARG group might have turned this competition mechanism in favor of NO production because it is known that the competitive inhibition of eNOS by asymmetric dimethylarginine can be reversed with supplemental L-arginine. ${ }^{21}$ This finding not only provides another possible mechanism for the normalization of endothelial function caused by L-arginine but also supports a substantial association between VEGF and eNOS in orchestrating the angiogenic process. Two recent studies in apolipoprotein E-hypercholesterolemic mice exhibiting attenuated collateral vessel formation in response to a FGF-2 disk angiogenesis system and hindlimb ischemia, respectively, ${ }^{22,23}$ showed that the angiogenic potential can be fully restored through the oral administration of L-arginine. Our study brings evidence that hypercholesterolemia-induced myocardial impairment of angiogenesis can also be overcome by L-arginine, closing in on the population of patients with advanced CAD who have thus far been poorly served by therapeutic angiogenesis. Considering the critical importance of NO release in the VEGFmediated angiogenic response, a role for eNOS and its precursor Akt in the molecular events involved in the al- tered angiogenic process associated with endothelial dysfunction is plausible and suggested in these patients.

In similar experimental designs comparing groups of pigs receiving the same diet regimen, we have previously shown that hypercholesterolemia-induced endothelial dysfunction significantly impairs both VEGF- and FGF-2mediated myocardial angiogenesis. ${ }^{9,24}$ It is believed that FGF-2-induced angiogenesis is dependent on Syndecan-4/ protein kinase $\mathrm{C} \alpha$ and relatively independent of Src kinases, in contrast to VEGF-induced angiogenesis, which invariably involves serine phosphorylation-activation of eNOS and the generation of NO in endothelial cells. ${ }^{25}$ Although this might result in a considerably different mechanistic link between the action of each of these growth factors and the local availability of NO, likely caused by key differences in their respective signal transduction pathways, endothelial dysfunction is the common denominator of growth factor-mediated angiogenesis inhibition in both of these studies. The model, however, obviously does not reproduce the multiple comorbid conditions observed in patients with CAD and the complex interactions by which they can impair endothelial function, and this constitutes an important limitation to our study. In this regard, however, it is interesting that the beneficial effects observed after Larginine administration are independent of modulations in the cholesterol levels and thus of any effect on the probable cause of endothelial dysfunction itself.

Taken together, these results suggest a paramount role for endothelial integrity in the success of protein growth factor-based therapeutic angiogenesis. This might explain the discrepancy observed to date between animal and clinical studies testing therapeutic angiogenesis and warrants the use not only of more appropriate research models displaying some degree of endothelial dysfunction but also of multimodal therapeutic approaches. To this end, the apparent capacity of eNOS to produce sufficient NO when adequately supplied with L-arginine indicates that the enzyme is at least partially functional, and combinations of growth factors with synthetic NO donors, eNOS activators, or antioxidants are other potentially interesting avenues.

\section{References}

1. Simons M, Bonow RO, Chronos NA, Cohen DJ, Giordano FJ, Hammond $\mathrm{HK}$, et al. Clinical trials in coronary angiogenesis: issues, problems, consensus: an expert panel summary. Circulation. 2000; 102:E73-86.

2. Henry TD, Annex BH, McKendall GR, Azrin MA, Lopez JJ, Giordano FJ, et al. The VIVA trial: vascular endothelial growth factor in ischemia for vascular angiogenesis. Circulation. 2003; 107:1359-65.

3. Sellke FW, Wang SY, Stamler A, Lopez JJ, Li J, Simons M. Enhanced microvascular relaxations to VEGF and bFGF in chronically ischemic porcine myocardium. Am J Physiol. 1996;271:H713-20.

4. Werns SW, Walton JA, Hsia HH, Nabel EG, Sanz ML, Pitt B. Evidence of endothelial dysfunction in angiographically normal coronary arteries of patients with coronary artery disease. Circulation. 1989;79:287-91. 
5. Bouloumie A, Schini-Kerth VB, Busse R. Vascular endothelial growth factor up-regulates nitric oxide synthase expression in endothelial cells. Cardiovasc Res. 1999;41:773-80.

6. Arnal JF, Yamin J, Dockery S, Harrison DG. Regulation of endothelial nitric oxide synthase mRNA, protein, and activity during cell growth. Am J Physiol. 1994;267:C1381-8.

7. Ziche M, Morbidelli L, Masini E, Amerini S, Granger HJ, Maggi CA, et al. Nitric oxide mediates angiogenesis in vivo and endothelial cell growth and migration in vitro promoted by substance P. J Clin Invest. 1994;94:2036-44.

8. Ziche M, Morbidelli L, Choudhuri R, Zhang HT, Donnini S, Granger HJ, et al. Nitric oxide synthase lies downstream from vascular endothelial growth factor-induced but not basic fibroblast growth factorinduced angiogenesis. J Clin Invest. 1997;99:2625-34.

9. Voisine P, Bianchi C, Ruel M, Malik T, Rosinberg A, Feng J, et al. Inhibition of the cardiac angiogenic response to exogenous vascular endothelial growth factor (VEGF) therapy in a porcine model of endothelial dysfunction. Surgery. 2004;136:407-15.

10. Wang BY, Singer AH, Tsao PS, Drexler H, Kosek J, Cooke JP. Dietary arginine prevents atherogenesis in the coronary artery of the hypercholesterolemic rabbit. J Am Coll Cardiol. 1994;23: 452-8.

11. Quyyumi AA, Dakak N, Diodati JG, Gilligan DM, Panza JA, Cannon RO. Effect of L-arginine on human coronary endotheliumdependent and physiologic vasodilation. J Am Coll Cardiol. 1997; 30:1220-7.

12. Lerman A, Burnett JC, Higano ST, McKinley LJ, Holmes DR. Longterm 1-arginine supplementation improves small-vessel coronary endothelial function in humans. Circulation. 1998;97:2123-8.

13. Tofukuji M, Metais C, Li J, Franklin A, Simons M, Sellke FW Myocardial VEGF expression after cardiopulmonary bypass and cardioplegia. Circulation. 1998;98(suppl):II242-8.

14. Sellke FW, Tofukuji M, Laham RJ, Li J, Hariawala MD, Bunting S, et al. Comparison of VEGF delivery techniques on collateral-dependent microvascular reactivity. Microvasc Res. 1998;55:175-8.

15. Ruel M, Sellke FW, Bianchi C, Khan TA, Faro R, Zhang JP, et al. Endogenous myocardial angiogenesis and revascularization using a gastric submucosal patch. Ann Thorac Surg. 2003;75:1443-9.

16. Cohen RA, Zitnay KM, Haudenschild CC, Cunningham LD. Loss of selective endothelial cell vasoactive functions caused by hypercholesterolemia in pig coronary arteries. Circ Res. 1988;63:903-10.

17. Hasdai D, Mathew V, Schwartz RS, Holmes DR Jr, Lerman A. The effect of basic fibroblast growth factor on coronary vascular tone in experimental hypercholesterolemia in vivo and in vitro. Coron Artery Dis. 1997;8:299-304.

18. Cai H, Harrison DG. Endothelial dysfunction in cardiovascular diseases: the role of oxidant stress. Circ Res. 2000;87:840-4.

19. Vallance P, Leone A, Calver A, Collier J, Moncada S. Endogenous dimethylarginine as an inhibitor of nitric oxide synthesis. J Cardiovasc Pharmacol. 1992;20(suppl 12):S60-2.

20. Bode-Boger SM, Boger RH, Kienke S, Junker W, Frolich JC. Elevated $\mathrm{L}$-arginine/dimethylarginine ratio contributes to enhanced systemic NO production by dietary 1-arginine in hypercholesterolemic rabbits. Biochem Biophys Res Commun. 1996;219:598-603.

21. Boger RH, Bode-Boger SM, Szuba A, Tsao PS, Chan JR, Tangphao O, et al. Asymmetric dimethylarginine (ADMA): a novel risk factor for endothelial dysfunction: its role in hypercholesterolemia. Circulation. 1998;98:1842-7.

22. Duan J, Murohara T, Ikeda H, Katoh A, Shintani S, Sasaki K, et al. Hypercholesterolemia inhibits angiogenesis in response to hindlimb ischemia: nitric oxide-dependent mechanism. Circulation. 2000;102 (suppl):III370-6.

23. Jang JJ, Ho HK, Kwan HH, Fajardo LF, Cooke JP. Angiogenesis is impaired by hypercholesterolemia: role of asymmetric dimethylarginine. Circulation. 2000;102:1414-9.

24. Ruel M, Wu GF, Khan TA, Voisine P, Bianchi C, Li J, et al. Inhibition of the cardiac angiogenic response to surgical FGF-2 therapy in a swine endothelial dysfunction model. Circulation. 2003;108(suppl 1):II335-40.
25. Cross MJ, Claesson-Welsh L. FGF and VEGF function in angiogenesis. Signaling pathways, biological responses and therapeutic inhibition. Trends Pharmacol Sci. 2001;22:201-7.

\section{Discussion}

Dr Harold L. Lazar (Boston, Mass). Have you considered using a statin instead of L-arginine? We and others have shown that pretreatment with statins in a similar model-we used a noncholesterol model-greatly protects and enhances the reaction and vasodilatation to endothelial function, so that in itself has a protective effect. Have you considered doing that?

Dr Voisine. This is a very insightful comment, and I thank you for it. We have indeed considered it, and we currently have groups of pigs receiving the hypercholesterolemic diet that are administered statins and that are planned to undergo a similar experimental protocol with growth factors. Interestingly, the L-arginine supplementation could overcome the endothelial dysfunction without affecting the cholesterol levels, which makes our model particularly attractive to study the combination of statins and growth factors.

Dr Lazar. It is a very interesting observation. But that is exactly what we saw, that statins, in the model of normal cholesterol levels, will actually improve endothelial function in the absence of any effect on endothelial function because, as you know, pigs by themselves do not make cholesterol. Therefore this is one of the pleiotrophic effects of statins, which I think would make it so good for this particular model.

Dr Robert C. Robbins (Stanford, Calif). We also have been taking our atorvastatin (Lipitor) every morning, so that is good.

How much arginine did you have to give? I did not see it in your abstract, or you did not mention how much you had to give.

Dr Voisine. We roughly gave $50 \mathrm{mg} / \mathrm{kg}$ twice daily.

Dr Robbins. Because, as you probably well know, I am sure Frank does, there is a cardiologist at Stanford who started a whole company on arginine bars. But the fact is, you have to give boatloads of this stuff to get any effect. Did you measure any levels?

Dr Voisine. We tried to measure the levels, and we did not observe any difference between the different treatment groups. This might be due to the very rapid turnover of L-arginine. We initially used a higher dose and had a few cases of aortic thrombi with hind-limb ischemia. We could not demonstrate a causal relationship between L-arginine administration and thrombus formation, but because it only occurred in that treatment group, we decreased the dose to $50 \mathrm{mg} / \mathrm{kg}$ twice daily and did not encounter other adverse events.

Dr Guo-Wei He (Hong Kong, China). VEGF has 2 major subtypes of receptors, KDR and FLT. I am wondering whether you have tested which subtype of VEGF receptors, KDR or FLT, mediates the effect in these microvessels.

Dr Voisine. That is another very interesting point. We used VEGF $_{165}$, and we are currently looking at the expression of both receptors. Unfortunately, it has been very difficult to perform adequate Western blot analyses thus far, and we are now studying the mRNA expression of both receptors of VEGF by means of Northern blotting. Our results are preliminary, but hopefully we will have answers to this question soon. 